\title{
A risk score to predict in-hospital mortality in patients with acute coronary syndrome at early medical contact: results from the Improving Care for Cardiovascular Disease in China-Acute Coronary Syndrome (CCC-ACS) Project
}

\author{
Peng Ran ${ }^{1 \#}$, Jun-Qing Yang ${ }^{1 \#}$, Jie Li ${ }^{1 \#}$, Guang Li $^{1}$, Yan Wang ${ }^{2}$, Jia Qiu ${ }^{1}$, Qi Zhong ${ }^{1}$, Yu Wang ${ }^{1}$, \\ Xue-Biao Wei ${ }^{1}$, Jie-Leng Huang ${ }^{1}$, Chung-Wah Siu ${ }^{3}$, Ying-Ling Zhou ${ }^{1}$, Dong Zhao ${ }^{4}$, Dan-Qing Yu ${ }^{1}$, \\ Ji-Yan Chen ${ }^{1}$; on behalf of the CCC-ACS Investigators
}

${ }^{1}$ Department of Cardiology, Guangdong Cardiovascular Institute, Guangdong Provincial Key Laboratory of Coronary Heart Disease Prevention, Guangdong Provincial People's Hospital, Guangdong Academy of Medical Sciences, Guangzhou, China; ${ }^{2}$ School of Public Health, Fudan University, Key Laboratory of Public Health Safety, Ministry of Education, Shanghai, China; ${ }^{3}$ Cardiology Division, Department of Medicine, The University of Hong Kong, Queen Mary Hospital, Hong Kong, China; ${ }^{4}$ Department of Epidemiology, Beijing Anzhen Hospital, Capital Medical University, Beijing Institute of Heart, Lung and Blood Vessel Diseases, Beijing, China

Contributions: (I) Conception and design: JY Chen, DQ Yu; (II) Administrative support: JY Chen, DQ Yu, JQ Yang; (III) Provision of study materials or patients: D Zhao; (IV) Collection and assembly of data: P Ran, J Li, J Qiu, Q Zhong, Y Wang, XB Wei, JL Huang; (V) Data analysis and interpretation: P Ran, JQ Yang, J Li, G Li, Y Wang, CW Siu, YL Zhou; (VI) Manuscript writing: All authors; (VII) Final approval of manuscript: All authors.

\#These authors contributed equally to this study.

Correspondence to: Ji-Yan Chen, MD; Dan-Qing Yu, MD. Department of Cardiology, Guangdong Cardiovascular Institute, Guangdong Provincial Key Laboratory of Coronary Heart Disease Prevention, Guangdong Provincial People’s Hospital, Guangdong Academy of Medical Sciences, Guangzhou 510080, China. Email: gdphospital@163.com; yudanqing2017@126.com.

Background: A number of models have been built to evaluate risk in patients with acute coronary syndrome (ACS). However, accurate prediction of mortality at early medical contact is difficult. This study sought to develop and validate a risk score to predict in-hospital mortality among patients with ACS using variables available at early medical contact.

Methods: A total of 62,546 unselected ACS patients from 150 tertiary hospitals who were admitted between 2014 and 2017 and enrolled in the Improving Care for Cardiovascular Disease in China-Acute Coronary Syndrome (CCC-ACS) project, were randomly assigned (at a ratio of 7:3) to a training dataset $(n=43,774)$ and a validation dataset $(n=18,772)$. Based on the identified predictors which were available prior to any blood test, a new point-based risk score for in-hospital death, CCC-ACS score, was derived and validated. The CCC-ACS score was then compared with Global Registry of Acute Coronary Events (GRACE) risk score.

Results: The in-hospital mortality rate was $1.9 \%$ in both the training and validation datasets. The CCCACS score, a new point-based risk score, was developed to predict in-hospital mortality using 7 variables that were available before any blood test including age, systolic blood pressure, cardiac arrest, insulin-treated diabetes mellitus, history of heart failure, severe clinical conditions (acute heart failure or cardiogenic shock), and electrocardiographic ST-segment deviation. This new risk score had an area under the curve (AUC) of 0.84 ( $\mathrm{P}=0.10$ for Hosmer-Lemeshow goodness-of-fit test) in the training dataset and 0.85 ( $\mathrm{P}=0.13$ for Hosmer-Lemeshow goodness-of-fit test) in the validation dataset. The CCC-ACS score was comparable to the Global Registry of Acute Coronary Events (GRACE) score in the prediction of in-hospital death in the validation dataset.

Conclusions: The newly developed CCC-ACS score, which utilizes factors that are acquirable at early medical contact, may be able to stratify the risk of in-hospital death in patients with ACS. 
Clinical trial registration: URL: http://www.clinicaltrials.gov. Unique identifier: NCT02306616.

Keywords: Acute coronary syndrome (ACS); in-hospital death; risk score; early medical contact

Submitted Dec 24, 2020. Accepted for publication Jan 21, 2021.

doi: $10.21037 / \mathrm{atm}-21-31$

View this article at: http://dx.doi.org/10.21037/atm-21-31

\section{Introduction}

Ischemic heart disease (IHD) is the leading cause of death globally $(1,2)$. In 2018 , the annual mortality ratio among Chinese patients with IHD exceeded 110/100,000, and it is steadily increasing (3). Acute coronary syndrome (ACS) is a severe manifestation of IHD with a prognosis that varies significantly among patients. Therefore, risk stratification is critical for decision-making and management implementation, such as timely invasive strategies for patients at high risk.

Several risk scores for ST-segment elevation myocardial infarction (STEMI), non-ST-segment elevation ACS (NSTE-ACS), and unselected ACS have been developed (4-8), among which some have been recommended by clinical guidelines (9-12). However, the existing risk score systems have some limitations (13). Firstly, most of them were developed prior to or during the early phase of the drug-eluting stent era, and minority of patients underwent percutaneous intervention, thus the discrimination power was relatively poor in those patients. Secondly, acquiring the variables for these risk scores is time consuming, which limits their utility at the point of early medical contact. Further, some risk scores at early medical contact were available, however some ACS patients at high risk were excluded in the registries developing risk score.

The present study aimed to develop and validate a simple and accurate risk score to predict in-hospital death in unselected patients with ACS at early medical contact by using data from the CCC-ACS registry, which represents the real-world practice in the drug-eluting stent era. We present the following article in accordance with the TRIPOD reporting checklist (available at http://dx.doi. org/10.21037/atm-21-31).

\section{Methods}

\section{Study protocol}

The CCC-ACS project design has been reported previously (14). Briefly, the American Heart Association (AHA) and Chinese Society of Cardiology (CSC) launched the CCC-ACS project in 2014 as a nationwide hospitalbased quality improvement registry program to improve the quality of care of patients with ACS. The present study was approval by the Ethics Committee of Beijing Anzhen Hospital, Capital Medical University. As the study used data from a retrospective registry, the requirement for informed consent was waived. All patient information was anonymized and de-identified before analysis. All procedures performed in this study involving human participants were in accordance with the Declaration of Helsinki (as revised in 2013).

\section{Study population and data collection}

From November 1, 2014 to June 30, 2017, CCC-ACS phases I and II enrolled 63,641 patients with ACS from 150 tertiary hospitals, which represented the highest level of medical care in the 7 geographical regions of China (Northern, Northeast, Eastern, Central, Southern, Southwest, and Northwest China).

Data were collected by trained data abstractors (medical doctors, nurses, medical postgraduates, and clinical research coordinators) at the participating hospitals through a webbased data collection platform (Oracle Clinical Remote Data Capture, Oracle). At each hospital, the first 20-30 ACS patients each month were consecutively enrolled. To ensure that consecutive cases were enrolled, quality audits were performed by third-party clinical research associates. The accuracy and completeness of the clinical data were verified using documents from approximately $5 \%$ of enrolled cases, who were randomly selected.

\section{Definitions}

Briefly, STEMI and non-ST-segment elevation myocardial infarction (NSTEMI) were defined according to the 2010 CSC STEMI guidelines (15) and the 2012 CSC NSTEACS guidelines (16), respectively. Unstable angina (UA) was defined as reported previously (14). Acute heart failure (AHF) and cardiogenic shock (CS) were defined according 
to the Chinese Guidelines for the Diagnosis and Treatment of Heart Failure 2014 (17), based on the patient's clinical condition recorded in the medical documentation on hospital admission. The endpoint was in-hospital death. Troponin I (TnI), troponin $\mathrm{T}(\mathrm{TnT})$, and creatine kinase $\mathrm{MB}$ isoenzyme (CK-MB) elevation was considered when the levels of these markers exceeded the upper level of normal (ULN) of the corresponding local laboratory. Estimated glomerular filtration rate (eGFR) was calculated according to the Modification of Diet in Renal Disease equation.

\section{Statistical analysis}

Statistical analyses were performed in SAS (version 9.4, SAS Institute, Cary, North Carolina). Data were presented as the mean \pm standard deviation (SD) for normally distributed data, or medians and interquartile ranges (IQR) for nonnormally distributed data. Normally and non-normally distributed variables were compared using Student's t-test and the Mann-Whitney U test, respectively. Categorical data were expressed as numbers (\%). Pearson's $\chi^{2}$ test or Fisher's exact test were used for categorical data, as appropriate. Using Proc Surveyselect (SAS, SAS Institute, Cary, North Carolina), the simple random sampling method was employed to randomly assign patients to a training dataset or a validation dataset at a ratio of 7:3. The CCC-ACS risk score was constructed by fitting demographic, medical history, clinical, and electrocardiographic variables, which were selected based their clinical significance and the findings of previous studies, as well as on their availability during early medical contact. Variables obtained by laboratory tests were not considered for entry into the model. Potential risk factors were screened through univariate logistic regression analysis with the level of significance set at $\mathrm{P}<0.05$. Independent predictors were identified by performing multivariate logistic regression analysis. Only variables with a $\mathrm{P}$ value of $<0.05$ in the multivariate analysis were entered into the final model. The integer score was generated by multiplying the $\beta$ coefficient of each selected variable by a constant and rounding the product to the nearest integer. Discrimination and calibration were assessed using the area under the receiver operating characteristic (ROC) curve (AUC) and the Hosmer-Lemeshow (H-L) goodness-of-fit test, respectively. Differences in the discriminatory power between the CCCACS score and the Global Registry of Acute Coronary Events (GRACE) score were evaluated using the $\chi^{2}$ test. All $\mathrm{P}$ values were 2 -tailed, and a $\mathrm{P}$ value of $<0.05$ was considered to represent statistical significance.

\section{Results}

There were 63,641 unselected ACS patients analyzed in this study, 44,549 patients initially assigned to the training dataset and 19,092 to the validation dataset. During the modeling process, $775(1.7 \%)$ and $320(1.7 \%)$ patients were excluded from the training and validation cohorts, respectively, due to having missing values for the finally incorporated variables (age, systolic blood pressure, cardiac arrest, and severe clinical conditions). The remaining 43,774 and 18,772 patients were enrolled in the final analyses (Figure 1).

In total, 1,181 in-hospital deaths occurred among the study patients, including $824(1.9 \%)$ in the training dataset and $357(1.9 \%)$ in the validation dataset. As shown in Table 1, except for prior dialysis ( $0.2 \%$ vs. $0.4 \%, \mathrm{P}=0.002)$, there were no significant differences in demographic, clinical, laboratory, electrocardiographic, or therapeutic characteristics, or in-hospital outcomes between the training and validation cohorts.

In the training dataset, the in-hospital death group had higher proportions of patients with STEMI, a history of heart failure, hypertension, diabetes mellitus, insulin-treated diabetes mellitus (ITDM), previous dialysis, ST-segment deviation, elevated CK-MB, and 5-fold elevated TNT or TNI. Furthermore, these patients were less likely to smoke or have a history of percutaneous coronary intervention (PCI). Patients in the in-hospital death group in the training dataset were also older, had higher heart rates and serum creatinine levels, and lower systolic blood pressure (SBP), diastolic blood pressure (DBP), and eGFR. Moreover, patients who died in hospital were more likely to present with cardiac arrest, AHF, and CS at admission (Table 2).

\section{Development and Validation of the CCC-ACS score}

The results of univariate and multivariate logistic regression analyses are displayed in Table S1. After univariable and multivariable selection, 7 variables emerged as predictors of mortality, including age, SBP, cardiac arrest, ITDM, history of heart failure, severe clinical conditions at admission (AHF and/ or CS), and ST-segment deviation. The scores assigned to each variable based on their estimated $\beta$ coefficients in the training dataset are shown in Table 3. The AUC for the original model was 0.84 , and the $\chi^{2}$ statistic for calibration was $11.48(\mathrm{P}=0.18)$.

The scores for each predictor based on their estimated $\beta$ coefficients are presented in Figure 2. The sum of the score which could theoretically range from 0 to 36 , could be used to estimate the risk of in-hospital death for individual patients. In 


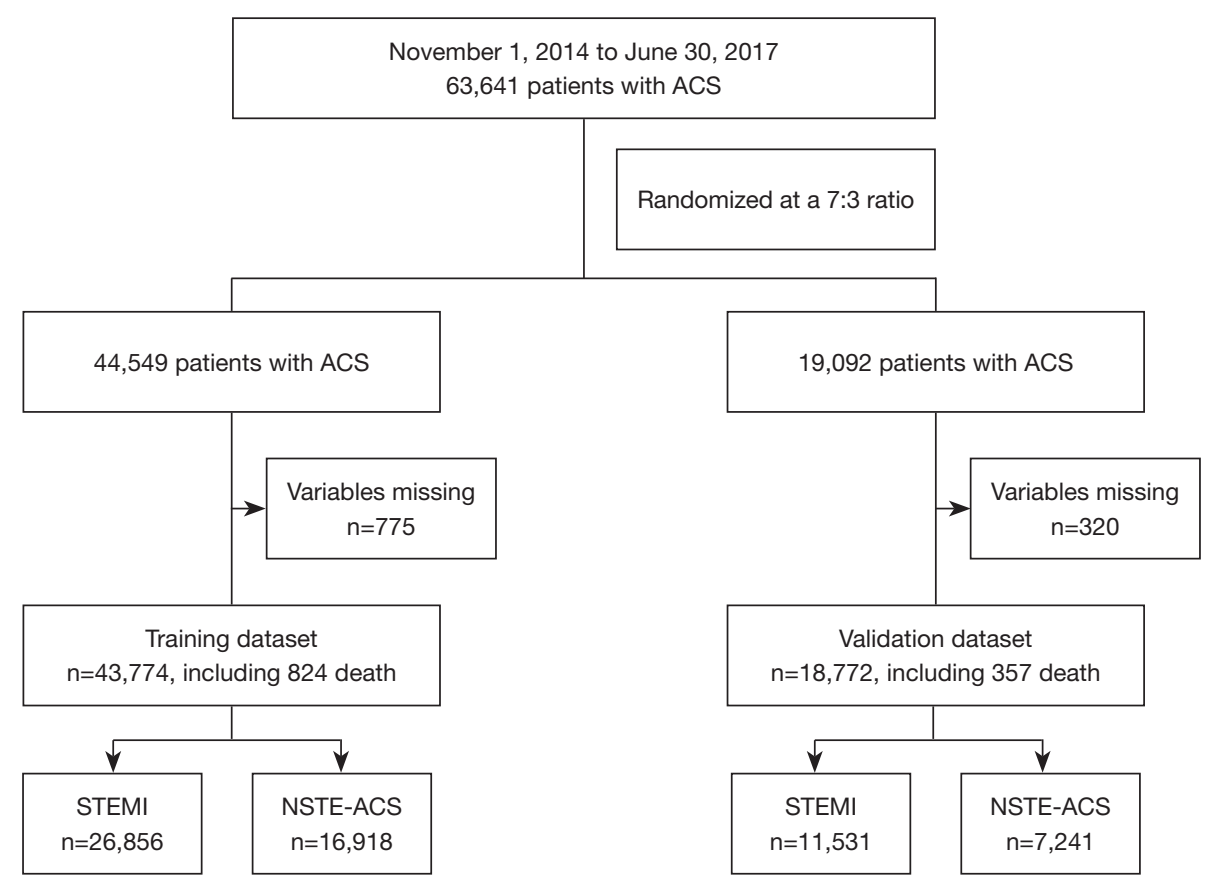

Figure 1 Study flow chart. The enrolled study population was divided into a training dataset and a validation dataset. ACS, acute coronary syndrome. STEMI, ST-segment elevation myocardial infarction. NSTE-ACS, non-ST-segment elevation acute coronary syndromes.

Table 1 Patient clinical characteristics

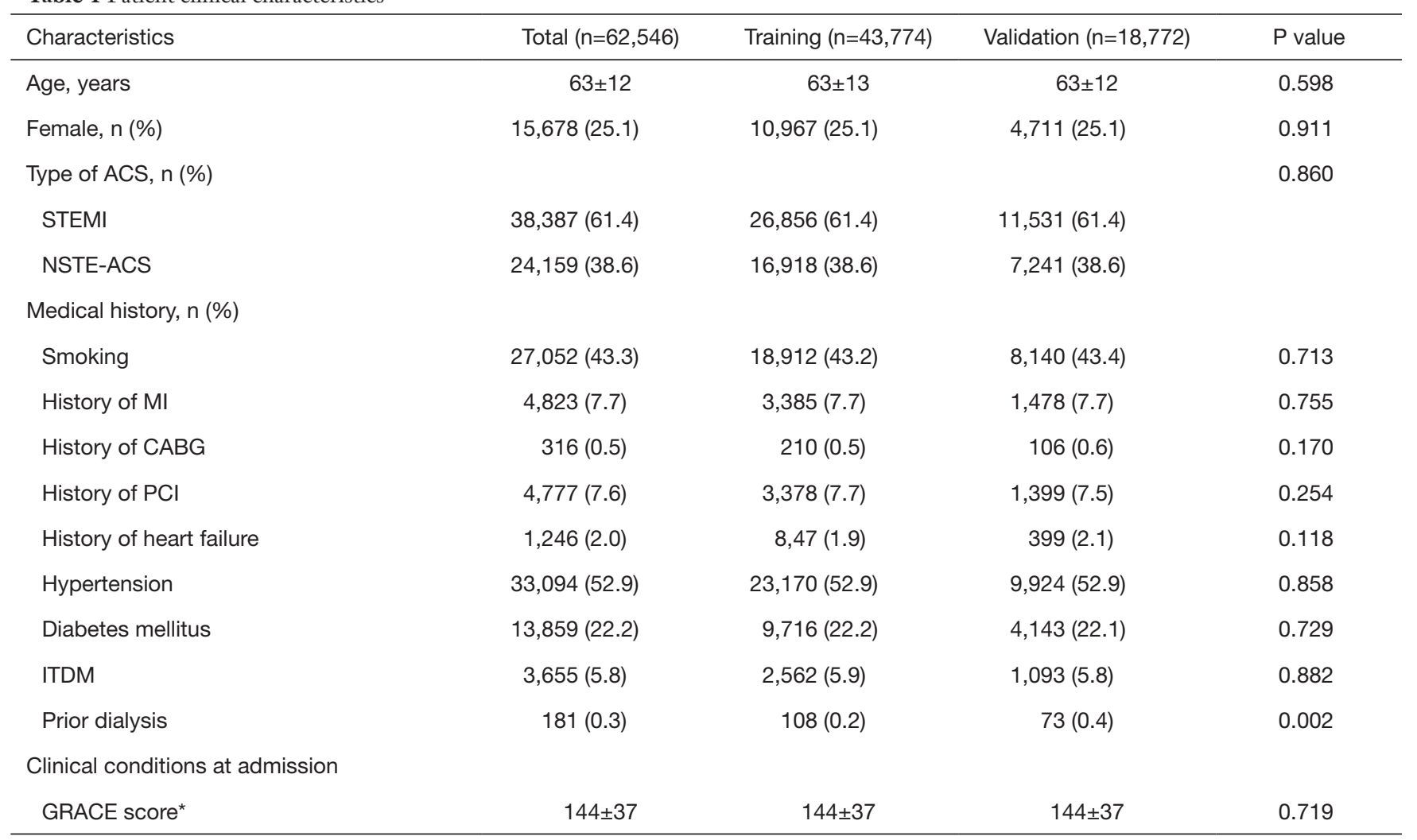

Table 1 (continued) 
Table 1 (continued)

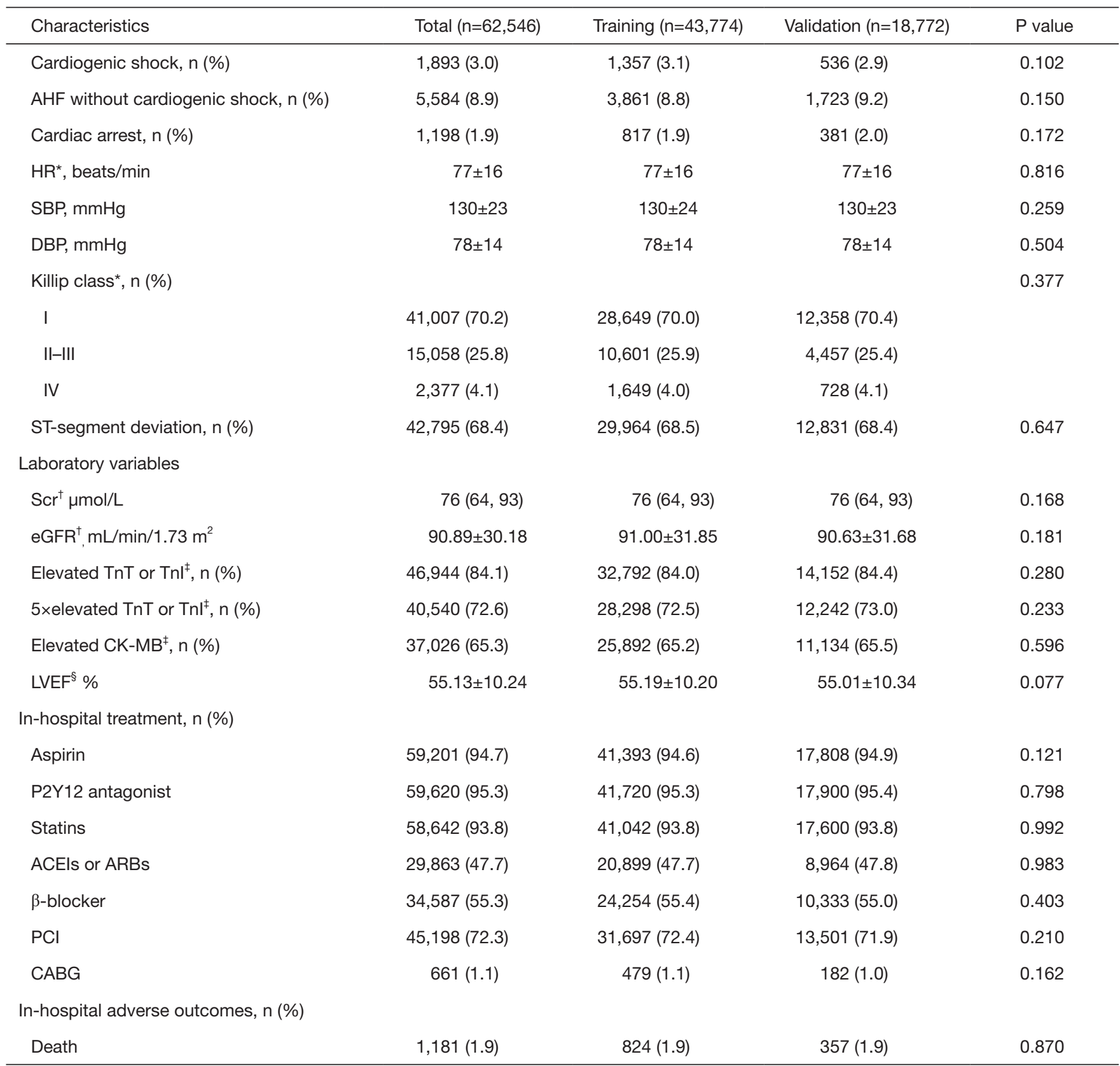

*, GRACE score and Killip class were not available for $11.8 \%(7,351 / 62,546)$ and $6.6 \%(4,104 / 62,546)$ of patients with ACS in the study population, respectively. HR was not available for 19 patients with ACS in the study population; ${ }^{\dagger}, 2.9 \%(1,836 / 62,546)$ of patients did not have Scr and $2.9 \%(1,836 / 62,546)$ of patients did not have eGFR in the study population; ${ }^{\ddagger}$, TnT or Tnl were not available for $10.8 \%(6,739 / 62,546)$ of patients with ACS, and elevated CK-MB were not available for $9.4 \%(5,856 / 62,546)$ of patients with ACS in the study population, respectively; ${ }^{\S}$, LVEF was not available for $22.8 \%(14,255 / 62,546)$ of patients with ACS in the study population. $\mathrm{ACEI}$, angiotensin-converting enzyme inhibitor; ACS, acute coronary syndrome; AHF, acute heart failure; ARBs, angiotensin receptor blockers; CABG, coronary artery bypass grafting; CK-MB, creatine kinase-MB; DBP, diastolic blood pressure; eGFR, estimated glomerular filtration rate; GRACE score, Global Registry of Acute Coronary Events risk score; HR, heart rate; ITDM, insulin-treated diabetes mellitus; LVEF, left ventricular ejection fraction; MI, myocardial infarction; NSTE-ACS, non-ST-segment elevation acute coronary syndromes; $\mathrm{PCl}$, percutaneous coronary intervention; SBP, systolic blood pressure; Scr, serum creatinine; STEMI, ST-segment elevation myocardial infarction. 
Table 2 Patient clinical characteristics in the training dataset

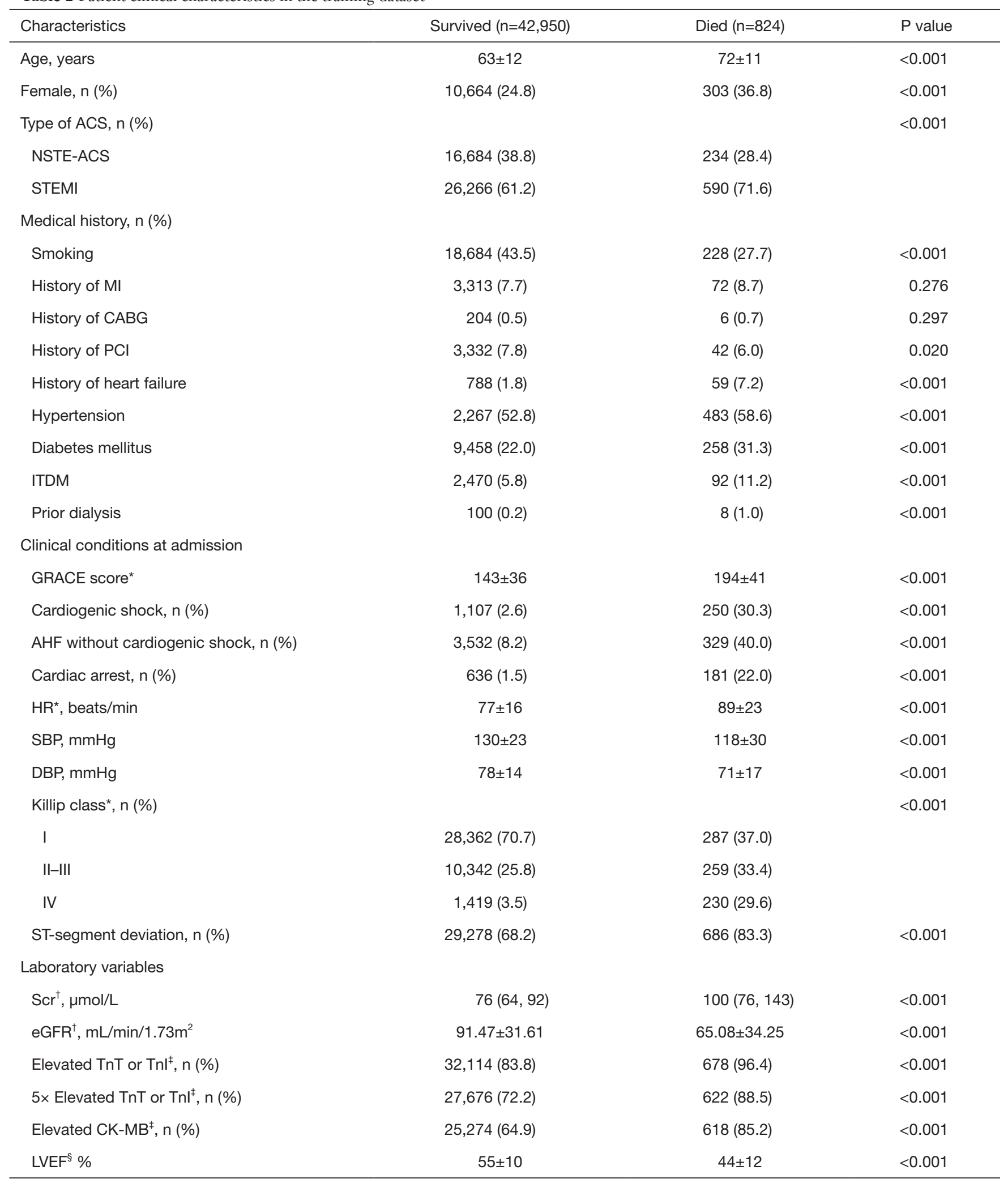

Table 2 (continued) 
Table 2 (continued)

\begin{tabular}{|c|c|c|c|}
\hline Characteristics & Survived $(n=42,950)$ & Died $(n=824)$ & $P$ value \\
\hline Aspirin & $40,701(94.8)$ & $692(84.0)$ & $<0.001$ \\
\hline P2Y12 antagonist & 41,007 (95.5) & $713(86.5)$ & $<0.001$ \\
\hline Statins & $40,407(94.1)$ & $635(77.1)$ & $<0.001$ \\
\hline$\beta$-blocker & $23,977(55.8)$ & 277 (33.6) & $<0.001$ \\
\hline $\mathrm{PCl}$ & $31,384(73.1)$ & $313(38.0)$ & $<0.001$ \\
\hline CABG & $406(0.9)$ & $73(8.9)$ & $<0.001$ \\
\hline
\end{tabular}

*, GRACE score and Killip class were not available for $11.7 \%(5,123 / 43,774)$ and $6.6 \%(2,875 / 43,774)$ of patients with ACS in the training dataset, respectively. HR was not available for 15 patients with ACS in the training dataset; ${ }^{\dagger}, 2.9 \%(1,257 / 43,774)$ of patients did not have Scr and $2.9 \%(1,257 / 43,774)$ of patients did not have eGFR in the training dataset; ${ }^{\ddagger}$, TnT or Tnl were not available for $10.8 \%(4,740 / 43,774)$ of patients with ACS, and elevated CK-MB were not available 9.3\% $(4,089 / 43,774)$ of patients with ACS in the training dataset; ${ }^{\S}$, LVEF was not available for $23.1 \%(10,102 / 43,774)$ of patients with ACS in the training dataset. ACEl, angiotensin-converting enzyme inhibitor; ACS, acute coronary syndrome; AHF, acute heart failure; ARBs, angiotensin receptor blockers; CABG, coronary artery bypass grafting; CK-MB, creatine kinase-MB; DBP, diastolic blood pressure; eGFR, estimated glomerular filtration rate; GRACE score, Global Registry of Acute Coronary Events risk score; HR, heart rate; ITDM, insulin-treated diabetes mellitus; LVEF, left ventricular ejection fraction; MI, myocardial infarction; NSTE-ACS, non-ST-segment elevation acute coronary syndromes; PCI, percutaneous coronary intervention; SBP, systolic blood pressure; Scr, serum creatinine; STEMI, ST-segment elevation myocardial infarction.

the training dataset, the actual obtained scores ranged from 0 to 31. The CCC-ACS score displayed good discrimination ability (AUC: 0.84 ) and calibration $\left(\chi^{2}=13.43, \mathrm{P}=0.10\right)$ (Figure $3 A$ ). In the validation dataset, the actual obtained scores ranged from 0 to 29, and the CCC-ACS score also displayed good discrimination ability (AUC: 0.85$)$ and calibration $\left(\chi^{2}=12.63\right.$, $\mathrm{P}=0.13$, Brier score $=0.02$ ) (Figure $3 B$ ).

Based on the obtained risk scores for in-hospital death, the training dataset was further categorized into the following 3 groups: low risk (score $\leq 12, \mathrm{n}=40,452$ ), moderate risk (score: $13-20, \mathrm{n}=2,919)$, and high risk (score $\geq 21, \mathrm{n}=403$ ). The event rate was $0.96 \%, 10.11 \%$, and $34.49 \%$, respectively (Figure 4 ). The validation dataset was also categorized into 3 groups: low risk (score $\leq 12, \mathrm{n}=17,323$ ), moderate risk (score: $13-20$, $\mathrm{n}=1,269$ ), and high risk (score $\geq 21, \mathrm{n}=180$ ). The event rate was $0.96 \%, 10.01 \%$, and $35.56 \%$, respectively (Figure 4).

\section{Performance in subgroups}

The CCC-ACS score also exhibited good discrimination ability after the patients were divided into subgroups according to sex, ACS type, and previous PCI or not (Table S2). After the exclusion of 2,228 patients who had missing values for GRACE variables, the remaining 16,544 patients in the validation dataset were used to compare the performances of the CCC-ACS score and the GRACE score. The 2 scores performed comparably in the prediction of in-hospital death (AUC: CCC-ACS 0.84, 95\% CI: 0.810.86 vs. GRACE $0.83,95 \%$ CI: $0.81-0.86, \mathrm{P}=0.69)$. The $\chi^{2}$ statistics for the CCC-ACS and GRACE scores were 5.12 $(\mathrm{P}=0.74)$ and $8.44(\mathrm{P}=0.39)$ respectively, showing the good calibration for in-hospital mortality.

\section{Discussion}

In the present study, a new in-hospital mortality risk score (CCC-ACS score) was developed and validated. The CCCACS risk score comprises 7 variables [age, cardiac arrest, ITDM, history of heart failure, severe clinical conditions at admission (AHF and/or CS), SBP, and ST-segment deviation], and demonstrated good discrimination ability and calibration in predicting the risk of in-hospital death for unselected ACS patients at early medical contact.

Several risk scores have been developed for risk stratification in patients with ACS. Among them, the Thrombolysis in Myocardial Infarction (TIMI) and GRACE scores are recommended by clinical guidelines and are widely applied in clinical practice. Both of these risk scoring systems can provide important information for predicting prognosis and determining the timing of interventions; 
Table 3 CCC-ACS risk sore final model

\begin{tabular}{|c|c|c|c|c|c|}
\hline Predictors & $\beta$ coefficient & $\chi^{2}$ & OR & $95 \% \mathrm{Cl}$ & $P$ value \\
\hline Cardiac arrest & 1.8500 & 244.94 & 6.36 & $5.05-8.02$ & $<0.0001$ \\
\hline ITDM & 0.6845 & 31.79 & 1.98 & $1.56-2.52$ & $<0.0001$ \\
\hline ST-segment deviation & 0.6148 & 39.37 & 1.85 & $1.53-2.24$ & $<0.0001$ \\
\hline No AHF or CS (reference) & - & - & - & - & - \\
\hline AHF without CS & 1.0462 & 103.74 & 2.85 & $2.33-3.48$ & $<0.0001$ \\
\hline CS & 1.9255 & 275.19 & 6.86 & $5.46-8.61$ & $<0.0001$ \\
\hline \multicolumn{6}{|l|}{ SBP } \\
\hline 80-99 & 0.7974 & 39.53 & 2.22 & $1.73-2.85$ & $<0.0001$ \\
\hline$<80$ & 1.1011 & 30.27 & 3.01 & $2.03-4.45$ & $<0.0001$ \\
\hline \multicolumn{6}{|l|}{ Age (years) } \\
\hline$<60$ (reference) & - & - & - & - & - \\
\hline $60-69$ & 0.6075 & 23.69 & 1.84 & $1.44-2.35$ & $<0.0001$ \\
\hline $70-79$ & 1.3572 & 134.96 & 3.89 & $3.09-4.89$ & $<0.0001$ \\
\hline $80-89$ & 1.8523 & 216.36 & 6.37 & $4.98-8.16$ & $<0.0001$ \\
\hline$\geq 90$ & 2.5142 & 108.12 & 12.36 & $7.69-19.85$ & $<0.0001$ \\
\hline
\end{tabular}

AHF, acute heart failure; CCC-ACS Risk Score: Improving Care for Cardiovascular Disease in China-Acute Coronary Syndrome Risk Score; CS, cardiogenic shock; ITDM, insulin-treated diabetes mellitus; SBP, systolic blood pressure.

however, they have some limitations (13). The TIMI risk score was derived from clinical trials and thus has inherent bias due to the exclusion of high-risk patients. The GRACE score was developed from a large-scale unbiased multicenter registry and was validated in external datasets; thus, it has an excellent performance when applied to the general population. Nevertheless, it has been found to lack accuracy for patients undergoing PCI (6), which may because less than $30 \%$ of patients in the GRACE (18) and Global Use of Strategies to Open Occluded Coronary Arteries IIB (GUSTO IIB) studies underwent PCI $(19,20)$. Furthermore, in the contemporary era, PCI has been used more widely, and its use has been accompanied by advances in medical treatments, such as P2Y12 antagonist, statin, angiotensin-converting enzyme inhibitors (ACEIs)/angiotensin receptor blockers (ARBs), and $\beta$-blockers. In the real-world registry used in the present study, which was compiled in the drug-eluting stent era, $72.3 \%$ of ACS patients underwent PCI. Therefore, an updated risk score that is fitting of current clinical practice is needed to supplement the use of previous scoring systems.

The CCC-ACS risk score shares 5 variables (age, cardiac arrest, SBP, severe clinical conditions at admission, and STsegment deviation) with previous risk scores $(4,21)$, and includes 2 (ITDM and history of heart failure) newly introduced variables. ITDM has been proven as a risk factor for adverse clinical outcomes in patients with NSTE-ACS or those undergoing PCI $(22,23)$. Patients with ITDM may have suffered a longer course of diabetes mellitus and may therefore represent a more severe disease condition (24). History of heart failure, another newly incorporated variable, has also been proved to be associated with in-hospital, 6-month, and 1-year mortality in ACS patients (25-28). A majority of previous studies have focused on AHF in patients with ACS, but a history of heart failure is also important and of independent value. ACS 
1. Find the score for each predictor

\begin{tabular}{|l|c|l|c|l|c|}
\hline Age, yrS & Points & SBP, $\mathrm{mmHg}$ & Points & Severe condition at admission & Points \\
\hline$<60$ & 0 & $\geq 140$ & 0 & No AHF or CS & 0 \\
$60 \sim 70$ & 2 & $100 \sim 140$ & 1 & AHF without CS & 4 \\
$70 \sim 80$ & 5 & $80 \sim 100$ & 3 & CS & 8 \\
$80 \sim 90$ & 7 & $<80$ & 4 & & \\
$\geq 90$ & 10 & & & & \\
\hline
\end{tabular}

\begin{tabular}{|lc|}
\hline Other Risk Factors & Points \\
\hline Cardiac arrest & 7 \\
History of heart failure & 2 \\
Insulin treated diabetic mellitus & 3 \\
ST-segment deviation & 2 \\
\hline
\end{tabular}

\section{Sum the score for all predictors}

3. Look up risk corresponding to total score

\begin{tabular}{|llllllll|}
\hline Total Points & $\leq 4$ & 8 & 12 & 16 & 20 & 24 & $>24$ \\
Probability of in-hospital & & & & & & & \\
mortality & $0.30 \%$ & $1.25 \%$ & $3.51 \%$ & $10.23 \%$ & $20.55 \%$ & $34.51 \%$ & $50.88 \%$ \\
\hline
\end{tabular}

Figure 2 Improving Care for Cardiovascular Disease in China-Acute Coronary Syndrome risk score (CCC-ACS score). SBP, systolic blood pressure. AHF, acute heart failure. CS, cardiogenic shock.
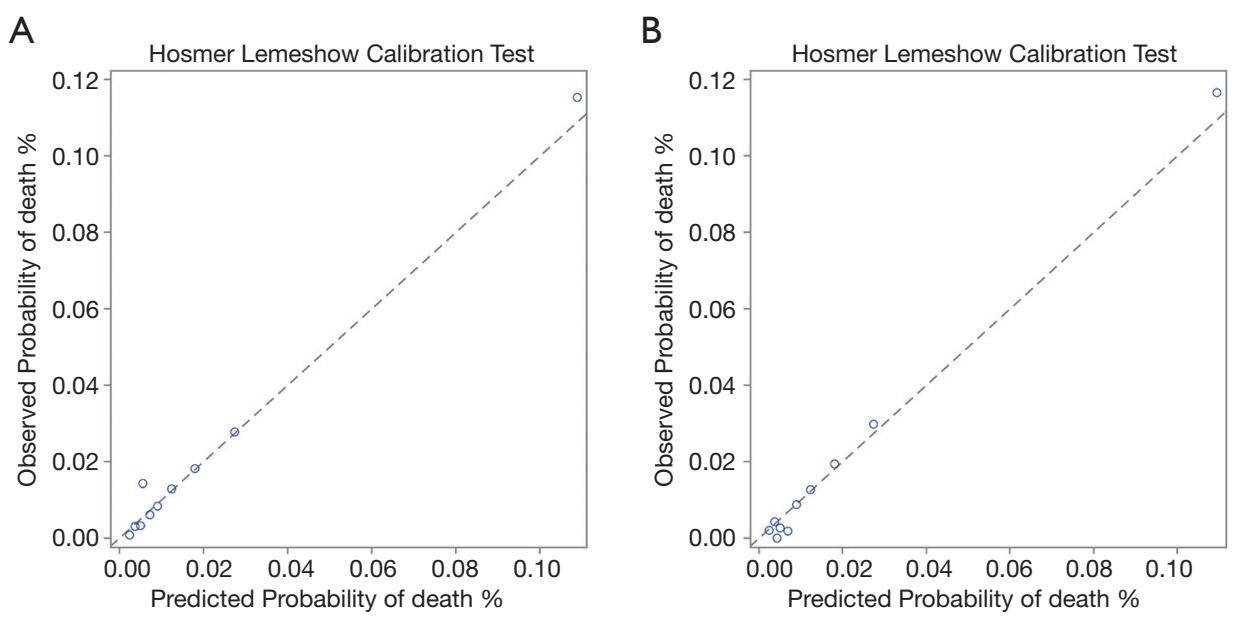

Figure 3 Calibration of Improving Care for Cardiovascular Disease in China-Acute Coronary Syndrome risk score (CCC-ACS score). (A) Calibration of CCC-ACS score in the training dataset. (B) Calibration of CCC-ACS score in the validation dataset. The diagonal line indicates perfect calibration.

patients with a history of heart failure may have lower cardiac reserve at baseline, and receive evidence-based therapies, such as $\beta$-blockers, ACEIs, and PCI, less frequently (25). Although some studies have associated a history of myocardial infarction with adverse outcomes $(29,30)$, it was not found to be an independent predictor after regression in the current analysis. This may be because, at least in part, a history of heart failure is correlated with and more powerful predictor than a history of myocardial infarction. Cardiac markers (TnI, TnT, and CK-MB) and serum creatinine have been demonstrated to be 


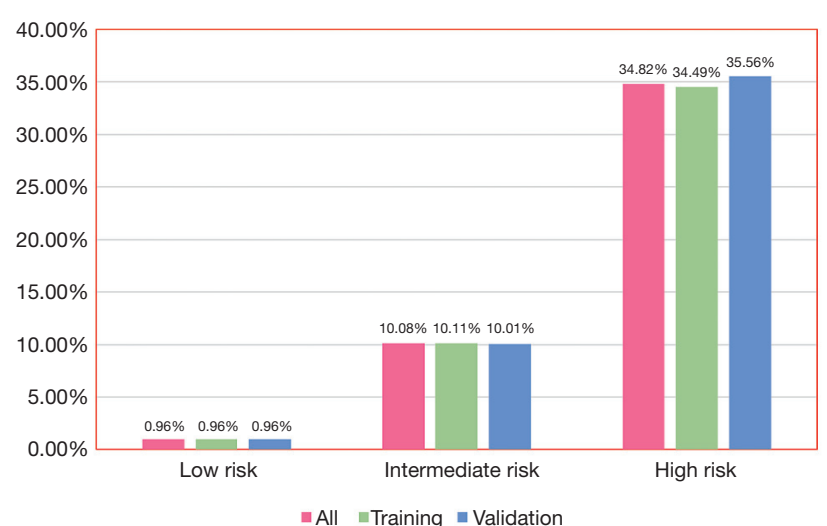

Figure 4 Observed incidence of in-hospital death. Observed incidence of in-hospital death according to categories of the Improving Care for Cardiovascular Disease in China-Acute Coronary Syndrome risk score (CCC-ACS score) in the training and validation datasets. low risk (score $\leq 12$ ), moderate risk (score: 13-20), and high risk (score $\geq 21$ ).

independently associated with adverse outcomes $(4,21,31,32)$, and can improve the discrimination ability of risk scores. However, these markers demand additional time and effort for blood tests to be performed; thus, they are usually not available during early medical contact. In fact, the data of cardiac markers and serum creatinine were lacking for a number of patients in the real-world registry used in the present study.

The main aim of this study was not to replace existing risk scores, but to establish a risk score with variables that are rapidly available at early medical contact. In the emergency unit, where it is busy and risk evaluation needs to be conducted promptly, a risk score based on readily available variables is practically more meaningful. This is also true for ambulance services, community health services, and other facilities with limited medical resources. Although it consists of rapidly obtainable variables, the CCC-ACS risk score displayed similar predictive ability for in-hospital death compared to the GRACE score. In addition, the CCC-ACS risk score exhibited good discrimination ability for those underwent PCI (AUC: 0.84), which is fitting of current clinical practice. Therefore, the CCC-ACS score may serve as a complement to previous risk scores.

There are potential applications of the CCC-ACS risk score. Firstly, stratifying patients at early medical contact without the need for blood tests may facilitate the quick identification of those with the highest risk and, subsequently, their quick and appropriate treatment. Secondly, some identified predictors in this model may provide useful information for updating other ACS risk scores.

\section{Limitations}

The present study has several limitations. Firstly, the rate of in-hospital mortality was relatively low among the patients in this study. One explanation was that phase I and phase II of the CCC-ACS project involved only tertiary hospitals, which exhibit a higher standard of patient care than other levels of hospitals. Furthermore, patients who died before or during transfer to the involved hospitals were not included in this study. Secondly, even though the CCC-ACS score was derived from a large-scale dataset, external validation is always required before its general application. Thirdly, the CCC-ACS project is a nationwide hospital-based quality improvement registry program without follow-up data. Therefore, whether the CCC-ACS risk score holds value for long-term prognosis is unknown. This question needs to be solved in further studies with follow-up. Finally, since the data in the CCC-ACS project were obtained from Chinese patients, further investigation is needed to determine whether the risk score performs as well in other populations.

\section{Conclusions}

The CCC-ACS CS score, which was developed from a largescale dataset of unselected ACS patients, can quantify the risk of in-hospital death for patients with ACS at early medical contact and may facilitate clinical decision-making. However, further external validation of this risk score is required.

\section{Acknowledgments}

We thank all participating hospitals for their contributions to the CCC-ACS project (Table S3).

Funding: The Improving Care for Cardiovascular Disease in China-Acute Coronary Syndrome (CCC-ACS) project is a collaborative program of the American Heart Association (AHA) and Chinese Society of Cardiology (CSC). The AHA was funded by Pfizer for the quality improvement initiative through an independent grant for learning and change. This work was also supported by Science and Technology Program of Guangzhou (No. 201704020124) and Sailing Foundation (Grant No. LHJJ201611011, LHJJ201612127), Beijing, China.

\section{Footnote}

Reporting Checklist: The authors have completed the 
TRIPOD reporting checklist. Available at http://dx.doi. org/10.21037/atm-21-31

Data Sharing Statement: Available at http://dx.doi. org/10.21037/atm-21-31

Conflicts of Interest: All authors have completed the ICMJE uniform disclosure form (available at http://dx.doi. org/10.21037/atm-21-31). Dr. DQY and Dr. PR report grants from Guangzhou Science and Technology Innovation Commission, during the conduct of the study; Dr. GL and Dr. JQY reports grants from Sailing Foundation, during the conduct of the study. The other authors have no conflicts of interest to declare.

Ethical Statement: The authors are accountable for all aspects of the work in ensuring that questions related to the accuracy or integrity of any part of the work are appropriately investigated and resolved. The present study was approval by the Ethics Committee of Beijing Anzhen Hospital, Capital Medical University. All procedures performed in this study involving human participants were in accordance with the Declaration of Helsinki (as revised in 2013). As the study used data from a retrospective registry, the requirement for informed consent was waived.

Open Access Statement: This is an Open Access article distributed in accordance with the Creative Commons Attribution-NonCommercial-NoDerivs 4.0 International License (CC BY-NC-ND 4.0), which permits the noncommercial replication and distribution of the article with the strict proviso that no changes or edits are made and the original work is properly cited (including links to both the formal publication through the relevant DOI and the license). See: https://creativecommons.org/licenses/by-nc-nd/4.0/.

\section{References}

1. Roth GA, Forouzanfar MH, Moran AE, et al. Demographic and epidemiologic drivers of global cardiovascular mortality. N Engl J Med 2015;372:1333-41.

2. Zhou M, Wang H, Zeng X, et al. Mortality, morbidity, and risk factors in China and its provinces, 1990-2017: a systematic analysis for the Global Burden of Disease Study 2017. Lancet 2019;394:1145-58.

3. Hu S, Gao R, Liu L, et al. Summary of the 2018 Report on Cardiovascular Diseases in China. Chinese Circulation Journal 2019;34:209-20.
4. Granger CB, Goldberg RJ, Dabbous O, et al. Predictors of hospital mortality in the global registry of acute coronary events. Arch Intern Med 2003;163:2345-53.

5. Antman EM, Cohen M, Bernink PJ, et al. The TIMI risk score for unstable angina/non-ST elevation MI: A method for prognostication and therapeutic decision making. JAMA 2000;284:835-42.

6. Palmerini T, Genereux P, Caixeta A, et al. A new score for risk stratification of patients with acute coronary syndromes undergoing percutaneous coronary intervention: the ACUITY-PCI (Acute Catheterization and Urgent Intervention Triage Strategy-Percutaneous Coronary Intervention) risk score. JACC Cardiovasc Interv 2012;5:1108-16.

7. Morrow DA, Antman EM, Giugliano RP, et al. A simple risk index for rapid initial triage of patients with STelevation myocardial infarction: an InTIME II substudy. LANCET 2001;358:1571-5.

8. Huynh T, Kouz S, Yan AT, et al. Canada Acute Coronary Syndrome Risk Score: a new risk score for early prognostication in acute coronary syndromes. Am Heart J 2013;166:58-63.

9. Ibanez B, James S, Agewall S, et al. 2017 ESC Guidelines for the management of acute myocardial infarction in patients presenting with ST-segment elevation: The Task Force for the management of acute myocardial infarction in patients presenting with ST-segment elevation of the European Society of Cardiology (ESC). Eur Heart J 2018;39:119-77.

10. Roffi M, Patrono C, Collet JP, et al. 2015 ESC Guidelines for the management of acute coronary syndromes in patients presenting without persistent ST-segment elevation: Task Force for the Management of Acute Coronary Syndromes in Patients Presenting without Persistent ST-Segment Elevation of the European Society of Cardiology (ESC). Eur Heart J 2016;37:267-315.

11. O'Gara PT, Kushner FG, Ascheim DD, et al. 2013 ACCF/ AHA guideline for the management of ST-elevation myocardial infarction: executive summary: a report of the American College of Cardiology Foundation/American Heart Association Task Force on Practice Guidelines. J Am Coll Cardiol 2013;61:485-510.

12. Amsterdam EA, Wenger NK, Brindis RG, et al. 2014 AHA/ACC Guideline for the Management of Patients with Non-ST-Elevation Acute Coronary Syndromes: a report of the American College of Cardiology/American Heart Association Task Force on Practice Guidelines. J Am Coll Cardiol 2014;64:e139-e228. 
13. Bawamia B, Mehran R, Qiu W, et al. Risk scores in acute coronary syndrome and percutaneous coronary intervention: a review. Am Heart J 2013;165:441-50.

14. Hao Y, Liu J, Liu J, et al. Rationale and design of the Improving Care for Cardiovascular Disease in China (CCC) project: A national effort to prompt quality enhancement for acute coronary syndrome. Am Heart J 2016;179:107-15.

15. Guideline for diagnosis and treatment of patients with STelevation myocardial infarction. Zhonghua Xin Xue Guan Bing Za Zhi 2010;38:675-90.

16. Guidelines for the diagnosis and treatment of non-STsegment elevation acute coronary syndromes. Chin J Cardiol 2012;40:353-67.

17. Chinese guidelines for the diagnosis and treatment of heart failure 2014. Zhonghua Xin Xue Guan Bing Za Zhi 2014;42:98-122.

18. Steg PG, Goldberg RJ, Gore JM, et al. Baseline characteristics, management practices, and in-hospital outcomes of patients hospitalized with acute coronary syndromes in the Global Registry of Acute Coronary Events (GRACE). Am J Cardiol 2002;90:358-63.

19. A comparison of recombinant hirudin with heparin for the treatment of acute coronary syndromes. N Engl J Med 1996;335:775-82.

20. Tamis-Holland JE, Palazzo A, Stebbins AL, et al. Benefits of direct angioplasty for women and men with acute myocardial infarction: results of the Global Use of Strategies to Open Occluded Arteries in Acute Coronary Syndromes Angioplasty (GUSTO II-B) Angioplasty Substudy. Am Heart J 2004;147:133-9.

21. Chin CT, Chen AY, Wang TY, et al. Risk adjustment for in-hospital mortality of contemporary patients with acute myocardial infarction: the acute coronary treatment and intervention outcomes network (ACTION) registry-get with the guidelines (GWTG) acute myocardial infarction mortality model and risk score. Am Heart J

2011;161:113-22.e2.

22. Palmerini T, Dangas G, Mehran R, et al. Predictors and implications of stent thrombosis in non-ST-segment elevation acute coronary syndromes: the ACUITY Trial. Circ Cardiovasc Interv 2011;4:577-84.

23. Ritsinger V, Saleh N, Lagerqvist B, et al. High event rate after a first percutaneous coronary intervention in patients with diabetes mellitus: results from the Swedish coronary angiography and angioplasty registry. Circ Cardiovasc Interv 2015;8:e002328.

24. Cosmi F, Shen L, Magnoli M, et al. Treatment with insulin is associated with worse outcome in patients with chronic heart failure and diabetes. Eur J Heart Fail 2018;20:888-95.

25. Zhang H, Goodman SG, Yan RT, et al. In-hospital management and outcomes of acute coronary syndromes in relation to prior history of heart failure. Eur Heart J Acute Cardiovasc Care 2016;5:214-22.

26. Ranasinghe I, Naoum C, Aliprandi-Costa B, et al. Management and outcomes following an acute coronary event in patients with chronic heart failure 1999-2007. Eur J Heart Fail 2012;14:464-72.

27. Sanchis J, Nunez J, Bodi V, et al. Influence of comorbid conditions on one-year outcomes in non-ST-segment elevation acute coronary syndrome. Mayo Clin Proc 2011;86:291-6.

28. Iakobishvili Z, Feinberg MS, Danicek V, et al. Prior heart failure among patients with acute coronary syndromes is associated with a higher incidence of in-hospital heart failure. Acute Card Care 2006;8:143-7.

29. Heggunje PS, Wade MJ, O'Rourke RA, et al. Early invasive versus ischaemia-guided strategies in the management of non-Q wave myocardial infarction patients with and without prior myocardial infarction; results of Veterans Affairs Non-Q Wave Infarction Strategies in Hospital (VANQWISH) trial. Eur Heart J 2000;21:2014-25.

30. Sutton AG, Finn P, Hall JA, et al. Predictors of outcome after percutaneous treatment for cardiogenic shock. Heart 2005;91:339-44.

31. Bagai A, Schulte PJ, Granger CB, et al. Prognostic implications of creatine kinase- $\mathrm{MB}$ measurements in STsegment elevation myocardial infarction patients treated with primary percutaneous coronary intervention. Am Heart J 2014;168:503-11.e2.

32. Galla JM, Mahaffey KW, Sapp SK, et al. Elevated creatine kinase- $\mathrm{MB}$ with normal creatine kinase predicts worse outcomes in patients with acute coronary syndromes: results from 4 large clinical trials. Am Heart J 2006;151:16-24.

Cite this article as: Ran P, Yang JQ, Li J, Li G, Wang Y, Qiu J, Zhong Q, Wang Y, Wei XB, Huang JL, Siu CW, Zhou YL, Zhao D, Yu DQ, Chen JY; on behalf of the CCC-ACS Investigators. A risk score to predict in-hospital mortality in patients with acute coronary syndrome at early medical contact: results from the Improving Care for Cardiovascular Disease in China-Acute Coronary Syndrome (CCC-ACS) Project. Ann Transl Med 2021;9(2):167. doi: 10.21037/atm-21-31 


\section{Supplementary}

Table S1 Logistic regression analysis for in-hospital mortality

\begin{tabular}{|c|c|c|c|c|c|c|}
\hline Predictors & \multicolumn{3}{|c|}{ Univariate analysis } & \multicolumn{3}{|c|}{ Multivariate analysis } \\
\hline Cardiac arrest & 19.03 & $15.91-22.76$ & $<0.0001$ & 6.36 & $5.05-8.02$ & $<0.0001$ \\
\hline History of heart failure & 4.14 & $3.16-5.42$ & $<0.0001$ & 1.61 & $1.18-2.20$ & 0.003 \\
\hline ITDM & 2.06 & $1.66-2.56$ & $<0.0001$ & 1.98 & $1.56-2.52$ & $<0.0001$ \\
\hline \multicolumn{7}{|c|}{ Clinical conditions at admission } \\
\hline No AHF or CS (reference) & - & - & - & - & - & - \\
\hline AHF without CS & 5.06 & $4.21-6.08$ & $<0.0001$ & 2.85 & $2.33-3.48$ & $<0.0001$ \\
\hline CS & 21.5 & $18.22-25.38$ & $<0.0001$ & 6.86 & $5.46-8.61$ & $<0.0001$ \\
\hline $100-139$ & 1.31 & $1.10-1.56$ & 0.002 & 1.38 & $1.15-1.65$ & $<0.001$ \\
\hline 80-99 & 4.05 & $3.26-5.03$ & $<0.0001$ & 2.22 & $1.73-2.85$ & $<0.0001$ \\
\hline$<80$ & 15.89 & $11.62-21.73$ & $<0.0001$ & 3.01 & $2.03-4.45$ & $<0.0001$ \\
\hline \multicolumn{7}{|l|}{ Age (years) } \\
\hline$<60$ (reference) & - & - & - & - & - & - \\
\hline $60-69$ & 1.93 & $1.53-2.43$ & $<0.0001$ & 1.84 & $1.44-2.35$ & $<0.0001$ \\
\hline $70-79$ & 4.25 & $3.43-5.26$ & $<0.0001$ & 3.89 & $3.09-4.89$ & $<0.0001$ \\
\hline 80-89 & 7.13 & 5.68-8.94 & $<0.0001$ & 6.37 & $4.98-8.16$ & $<0.0001$ \\
\hline
\end{tabular}

AHF, acute heart failure; CS, cardiogenic shock; ITDM, insulin-treated diabetes 
Table S2 Model performance in subgroups

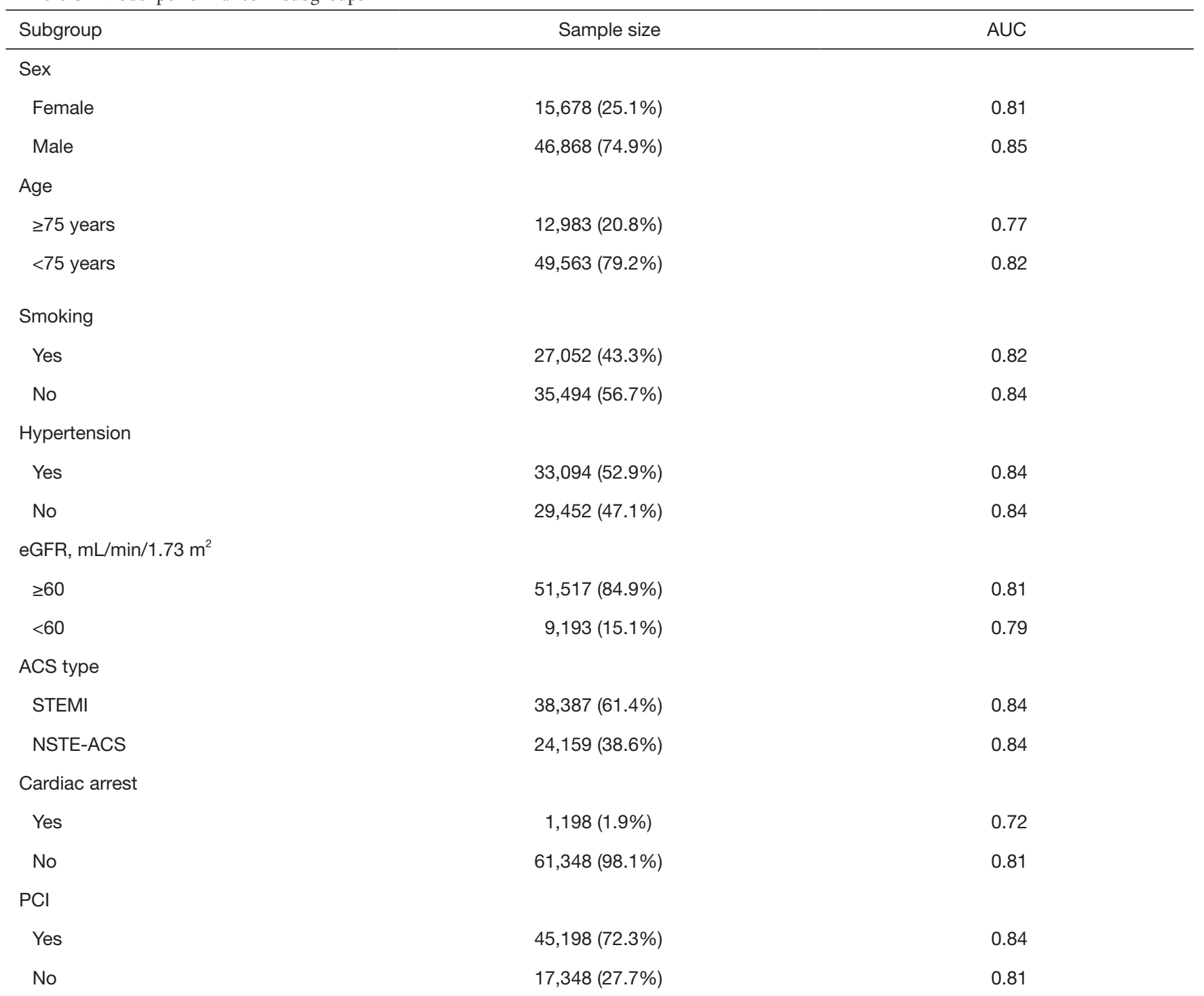

ACS, acute coronary syndrome; AHF, acute heart failure; AUC, area under the curve; CS, cardiogenic shock; eGFR, estimated glomerular filtration rate; NSTE-ACS, non-ST-segment elevation acute coronary syndromes; STEMI, ST-segment elevation myocardial infarction. 
Table S3 Investigators of CCC-ACS project

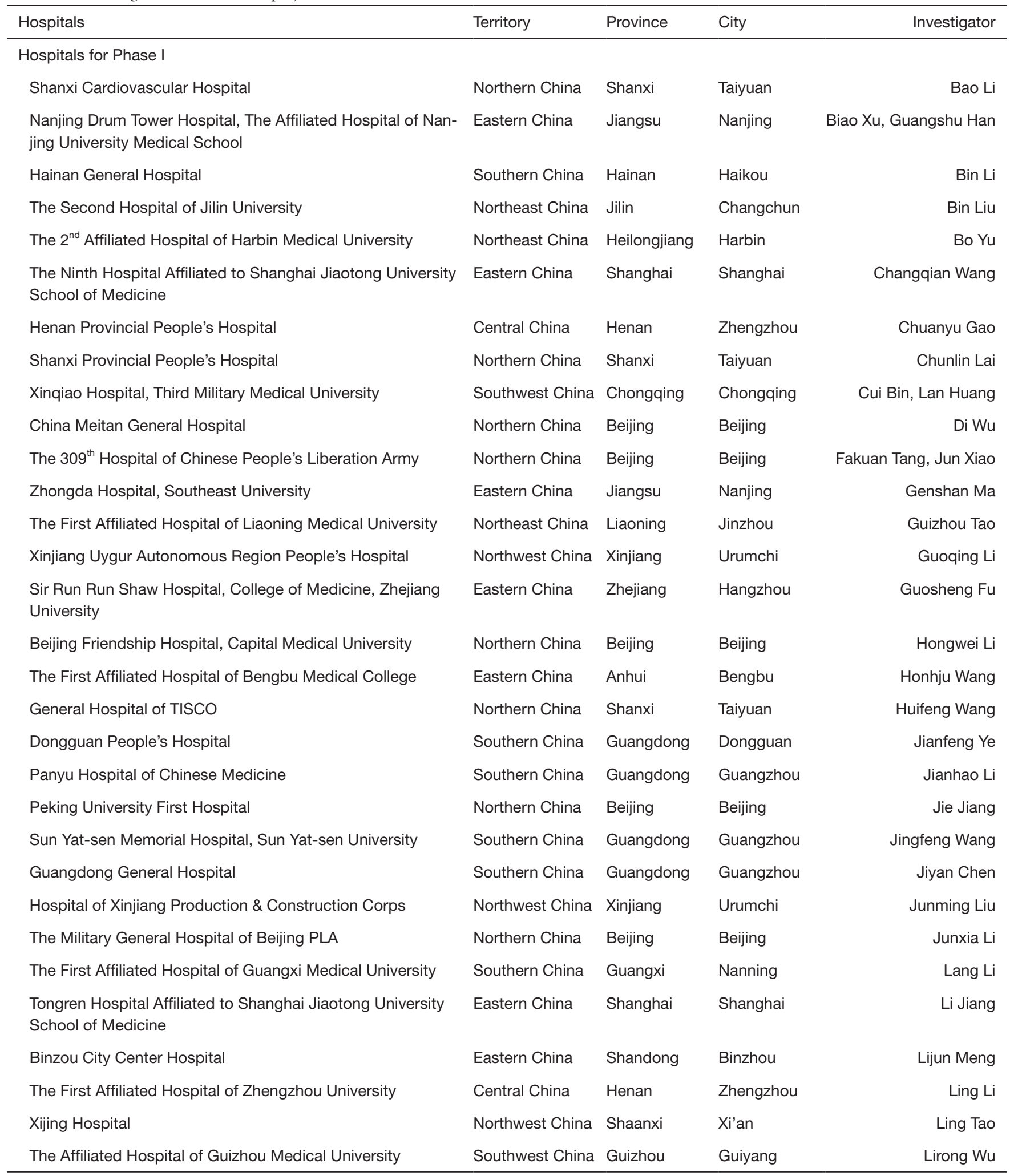

Table S3 (continued) 
Table S3 (continued)

\begin{tabular}{|c|c|c|c|c|}
\hline Hospitals & Territory & Province & City & Investigator \\
\hline Haikou People's Hospital & Southern China & Hainan & Haikou & Moshui Chen \\
\hline Chenzhou First People's Hospital & Central China & Hunan & Chenzhou & Qiaoqing Zhong \\
\hline People's Hospital of Qinghai Province & Northwest China & Qinghai & Xining & Rong Chang \\
\hline Affiliated Hospital of Ningxia Medical University & Northwest China & Ningxia & Yinchuan & Shaobin Jia \\
\hline North Jiangsu People's Hospital & Eastern China & Jiangsu & Yangzhou & Shenghu $\mathrm{He}$ \\
\hline Shanghai Sixth People's Hospital & Eastern China & Shanghai & Shanghai & Shixin Ma \\
\hline The First Hospital of Handan & Northern China & Hebei & Handan & Shuanli Xin \\
\hline Huai'an First People's Hospital & Eastern China & Jiangsu & Huai'an & Shuren Ma \\
\hline The First Affiliated Hospital of Chongqing Medical University & Southwest China & Chongqing & Chongqing & Suxin Luo \\
\hline Navy General Hospital & Northern China & Beijing & Beijing & Tianchang Li \\
\hline Zhejiang Provincial Hospital of TCM & Eastern China & Zhejiang & Hangzhou & Wei Mao \\
\hline The Third Xiangya Hospital of Central South University & Central China & Hunan & Changsha & Weihong Jiang \\
\hline Hebei General Hospital & Northern China & Hebei & Shijiazhuang & Xiaoyong Qi \\
\hline Inner Mongolia People's Hospital & Northern China & Inner Mongolia & Hohhot & Xingsheng Zhao \\
\hline The General Hospital of Shenyang Military Region & Northeast China & Liaoning & Shenyang & Yaling Han \\
\hline The First Hospital of Jilin University & Northeast China & Jilin & Changchun & Yang Zheng \\
\hline Tianjin Chest Hospital & Northern China & Tianjin & Tianjin & Yin Liu \\
\hline Hunan Provincial People's Hospital & Central China & Hunan & Changsha & Ying Guo \\
\hline People's Hospital of Yuxi City & Southwest China & Yunnan & Yuxi & Yinglu Hao \\
\hline The People's Hospital of Guangxi Zhuang Autonomous Region & nSouthern China & Guangxi & Nanning & Yingzhong Lin \\
\hline The First Teaching Hospital of Xinjiang Medical University & Northwest China & Xinjiang & Urumchi & Yitong Ma \\
\hline Baogang Hospital & Northern China & Inner Mongolia & Baotou & Yongdong $\mathrm{Li}$ \\
\hline Tianjin Medical University General Hospital & Northern China & Tianjin & Tianjin & Yuemin Sun \\
\hline
\end{tabular}

Table S3 (continued) 
Table S3 (continued)

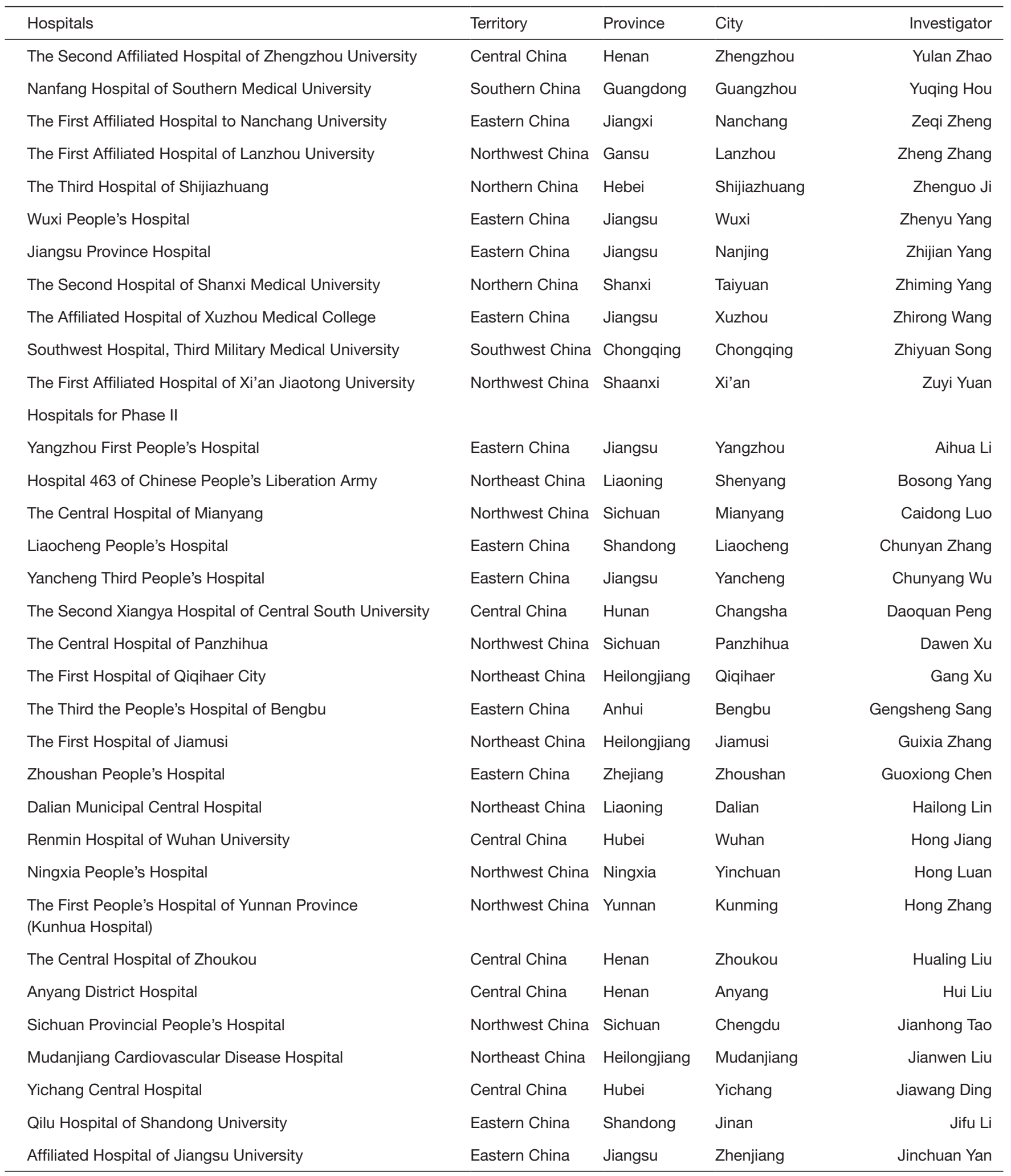

Table S3 (continued) 
Table S3 (continued)

\begin{tabular}{|c|c|c|c|c|}
\hline Hospitals & Territory & Province & City & Investigator \\
\hline The First Affiliated Hospital of Fujian Medical University & Eastern China & Fujian & Fuzhou & Jinzi Su \\
\hline Chengdu Third People's Hospital & Northwest China & Sichuan & Chengdu & Jiong Tang \\
\hline Yantaishan Hospital & Eastern China & Shandong & Yantai & Juexin Fan \\
\hline Zhongshan Hospital Affiliated to Fudan University & Eastern China & Shanghai & Shanghai & Junbo Ge \\
\hline Longyan First Hospital & Eastern China & Fujian & Longyan & Kaihong Chen \\
\hline Affiliated Hospital of Guangdong Medical College & Southern China & Guangdong & Guangzhou & Keng Wu \\
\hline Jiangxi Provincial People's Hospital & Eastern China & Jiangxi & Nanchang & Lang Ji \\
\hline The First Hospital of Haerbin City & Northeast China & Heilongjiang & Harbin & Lin Wei \\
\hline Central Hospital Affiliated to Shenyang Medical College & Northeast China & Liaoning & Shenyang & $\begin{array}{r}\text { Man Zhang, Kaiming } \\
\text { Chen }\end{array}$ \\
\hline The Central Hospital of Wuhan & Central China & Hubei & Wuhan & Manhua Chen \\
\hline Hangzhou First People's Hospital & Eastern China & Zhejiang & Hangzhou & Ningfu Wang \\
\hline The Central Hospital of Xuzhou & Eastern China & Jiangsu & Xuzhou & Peiying Zhang \\
\hline The Second Hospital of Dalian Medical University & Northeast China & Liaoning & Dalian & Peng Qu \\
\hline \multicolumn{2}{|c|}{$\begin{array}{l}\text { The First Affiliated Hospital of Liaoning University of TraditionalNortheast China } \\
\text { Chinese Medicine }\end{array}$} & Liaoning & Shenyang & Ping Hou \\
\hline Wuzhou People's Hospital & Southern China & Guangxi & Wuzhou & Shaowu Ye \\
\hline The Central Hospital of Jilin & Northeast China & Jilin & Changchun & Shuangbin Li \\
\hline Xiangya Hospital Central South University & Central China & Hunan & Changsha & Tianlun Yang \\
\hline Guangzhou Red Cross Hospital & Southern China & Guangdong & Guangzhou & Tongguo Wu \\
\hline The First Affiliated Hospital of Guangzhou Medical College & Southern China & Guangdong & Guangzhou & Wei Wang \\
\hline The First Affiliated Hospital of Wenzhou Medical University & Eastern China & Zhejiang & Wenzhou & Weijian Huang \\
\hline The Second Affiliated Hospital of Soochow University & Eastern China & Jiangsu & Suzhou & Weiting Xu \\
\hline Wuhan Asia Heart Hospital & Central China & Hubei & Wuhan & Xi Su \\
\hline The First Affiliated Hospital of Soochow University & Eastern China & Jiangsu & Suzhou & Xiangjun Yang \\
\hline Affiliated Hospital of Yan'an University & Northwest China & Shaanxi & Yan'an & Xiaochuan Ma \\
\hline The First People's Hospital of Jining & Eastern China & Shandong & Jining & Xiaofei Sun \\
\hline
\end{tabular}

Table S3 (continued) 
Table S3 (continued)

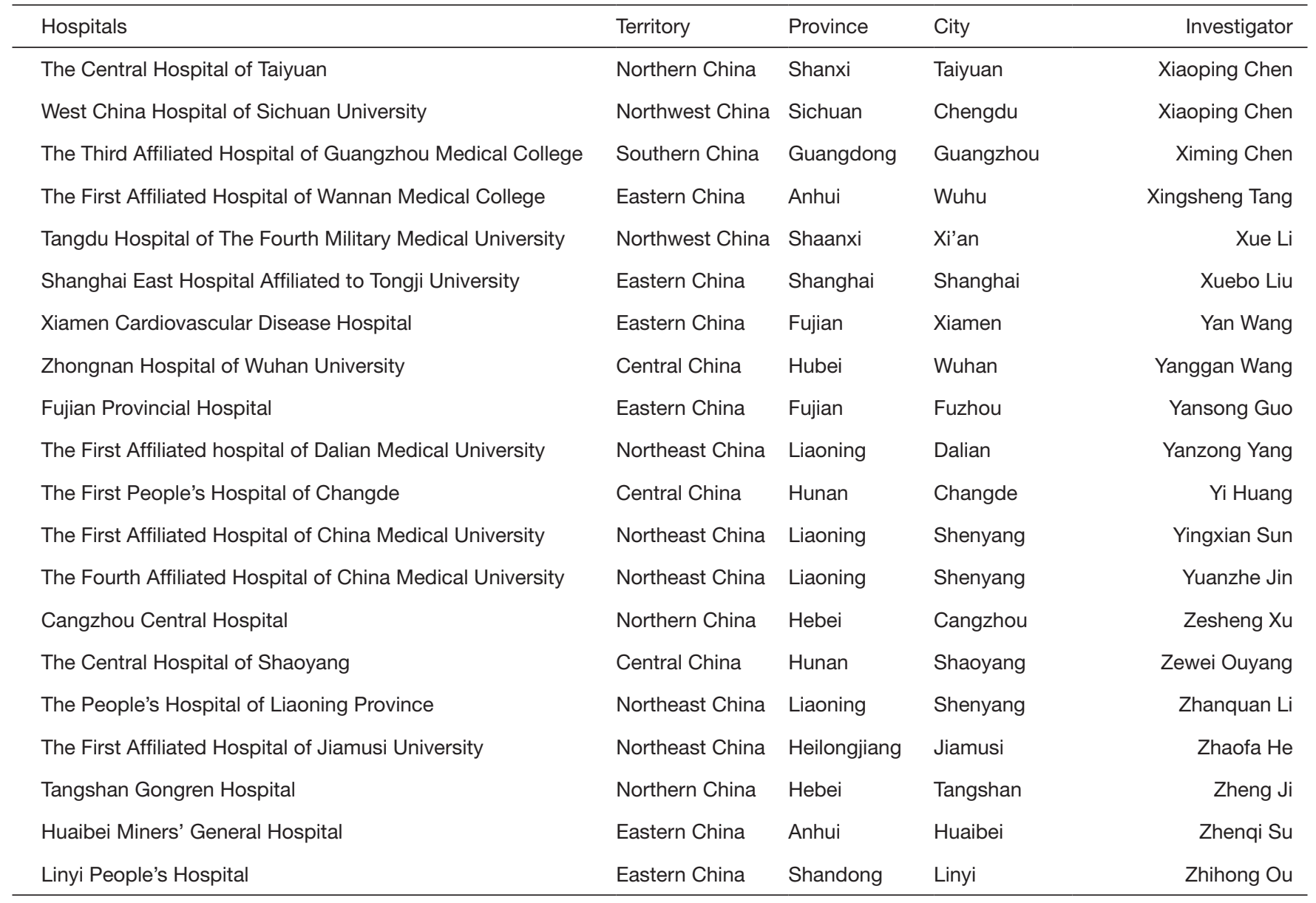

